\title{
Die stratigraphische Stellung des Warthe-Stadiums in Polen
}

\author{
Von S. Z. RozYcki, Warszawa
}

\author{
Mit 4 Abbildungen im Text
}

$\mathrm{Z}$ us a mmenf a ssung. Nach P. Woldstedt ist Mittelpolen das klassische Gebiet des Warthe-Stadiums. Seine stratigraphische Stellung determinieren folgende Tatsachen:

1. Die Lage der Frontalmoränen des Warthe-Stadiums, 50-120 km nördlich vom maximalen Bereich der Mittelpolnischen Vereisung (Riß, Saale-Vereisung, Radomka-Stadium), deren Geschiebemergel die organogenen Sedimente des vorletzten Interglazials (M/R, Holstein) bedecken.

2. Vollständige, paläobotanisch genau untersuchte Serien des letzten Interglazials (R/W) auf der Moränenoberfläche des Warthe-Stadiums.

3. Das Moränenniveau des Warthe-Stadiums ist von dem des maximalen Bereichs der Mittelpolnischen Vereisung (Radomka-Stadium) durch eine Periode getrennt, während der sich eine Tiefenerosion entwickelt hat, die die Flußtäler bis $15-20 \mathrm{~m}$ austiefte. Dann wurden sie mit Sedimenten eines alluvialen Akkumulationszyklus gefüllt (Pilica-Interstadium), die mit Warwenton oder in anderen Stellen mit einer Lößschicht bedeckt waren. Diese Sedimentserie kann man bis ca. $100 \mathrm{~km}$ nördlich vom maximalen Bereich des Warthe-Stadiums beobachten.

4. Eine ähnliche Sedimentserie wie im vorhergehenden Interstadium kann man nach dem Warthe-Stadium im Sektor $20^{\circ}-23^{\circ} \mathrm{E}$ in der Umgegend Warschaus und am unteren Bug feststellen. Sie gehört zum Bug/Narew-Interstadium. Später findet noch eine Transgression des Inlandeises (Wkra-Stadium) und ein Interstadium statt (Regimin-Interstadium), in das die letzte Transgression der vorletzten Vereisung des Mława-Stadiums einrückt.

5. Vollständige Sedimentserien des letzten Interglazials (R/W; Eem) erscheinen in Senken nach den oben erwähnten Stadien (mit einbegriffen das Mława-Stadium). Nördlich vom Gebiet der Mława-Moränen sind die Interglaziale dieses Alters schon mit Moränen der letzten Vereisung bedeckt (W; Weichsel-Vereisung, Baltische Vereisung).

6. Fossile Podsol-Böden des Pilica-Interstadiums, die von Warthe-Moränen bedeckt sind, kennt man aus der Gegend der unteren Pilica.

Unbekannt sind dagegen noch die organogenen Sedimentserien, die zwischen den Moränenniveaus des Radomka- und Warthe-Stadiums liegen. Höchstwahrscheinlich gehören zu dieser Zeit die Torfe, die in Brzozowica bei Bezdzin gefunden wurden, und die ca. $80 \mathrm{~km}$ südlich vom maximalen Bereich des Warthe-Stadiums liegen. Das palynologische Diagramm aus Brzozowica hat interstadiale Merkmale, obgleich es den Gedanken nahelegt, daß dort im Optimum dichte Kiefer-FichtenWälder wuchsen.

Auf Grund dieser Tatsachen ist der Verfasser der Ansicht, daß das Warthe-Stadium eins von den drei Stadien aus der Rezessionsperiode der vorletzten Vereisung ist. Sie waren durch deutliche Interstadien getrennt, die ähnlichen klimatischen Schwankungen unterlagen.

Die Verbreitungsgrenze des baltischen braunen Porphyrs, die nach V. und K. MiLthers den Bereich ihrer "Warthe-Vereisung " determiniert, zeigt in Mittelpolen keine Übereinstimmung mit dem Verlauf der Frontalmoränen, und im Sektor $19^{\circ}-22^{\circ} \mathrm{E}$ rückt sie bis zum Rand der SaaleVereisung. Westlich und östlich von diesem Sektor entspricht die Verteilung des braunen Porphyrs dem Bereich des Warthe-Stadiums. Die großen Mengen der lokalen Feuersteine aus der Jura- und Kreideformation in den Moränen der älteren Stadien machen die Anwendung der Methode des Geschiebekoeffizienten Konrad RICHTER's unmöglich.

$\mathrm{Sum} \mathrm{m}$ a ry. The stratigraphic position of the "Varta Stadium" is defined by the following facts in Middle Poland: 1) End-moraines of the "Varta Stadium" are located 50-120 km. northward from maximal extension (Radomka Stadium) of the Middle polish glaciation ( $\mathrm{R}$, Saale glac.); these moraine deposits cover organogenic deposits of the penultimate interglaciation $(M / R$; Holstein Intg1.). 2) Last interglacial deposits are known from many places in depressions without outflow and which are distributed on the surface of moraines from Varta stadium. 3) The morainic horizon of the Varta stadium is separated from the one of the maximal extension of the Middle Polish glaciation by erosive valleys $15-20 \mathrm{~m}$. deep and filled with deposits representing one cycle of fluvial accumulation (Pilica interstadial). These deposits, covered by varved clays or in other places by loess, may be traced as far as $100 \mathrm{~km}$. north from the maximal reach of the "Varta Stadium". 4) The "Varta Stadium" was followed (in the region between $20^{\circ}-23^{\circ} \mathrm{E}$ ) by the Bug Narev Interstadial, then by the ice advance of the Vkra Stadium followed by an additional interstadial (Regimin Interstadial) and then by the last advance of the penultimate glaciation - Młava 
stadium. Northward from the area where the "Młava Stadium" deposits occur on the surface, interglacial series of that age are covered by morainic deposits of the last glaciation (W, Weichsel glac., Baltic glac.). 6) The fossil soils of podzol type, which are connected with Pilica Interstadial and covered by moraines of the Varta Stadium, are known in lower Pilica region. But organogenic deposits of that age, separating the Radomka and Varta Stadium (morainic horizons!) are not known until now. Peats found at Brzozowica near Bedzin some $80 \mathrm{~km}$. south of the maximal extension of the Varta Stadium which show palynological features of an interstadial character, are most probably of that age.

The Varta Stadium represents in writer's opinion one of the three recessional stadia of the penultimate glaciation, which are separated by well marked interstadials corresponding to climatic oscillations of similar order of magnitude.

The limit of occurrence of the brown Baltic porphyry, which was regarded by V. and K. Milthers as a dead line of their "Varta Glaciation", runs discordantly with the endmorainic zones of that age through Middle Poland. In the region between $19^{\circ}-22^{\circ}$ it is shifted southward, up to the maximal reach of the Middle Polish glaciation. To the west and to the east from the mentioned region the occurrence of the brown porphyry is coextensive with the reach of the Varta stadium. The large quantities of local Jurassic and Cretaceous flints (to $80 \%$ within $0,5-5,0 \mathrm{~cm}$. grain size) in moraines of older stadia make it impossible to apply K. RichteR's boulder coefficient method.

Die in den letzten Jahren durchgeführten Untersuchungen im Pleistozän Polens haben eine eingehende Bearbeitung der Stratigraphie des anaglazialen Teiles des vorletzten Interglazials (Mindel/Riß, Holstein, Masovien I) und der mittelpolnischen Vereisung (Riß, Saale-Eiszeit) ermöglicht.

Dadurch tritt ziemlich deutlich die Stellung des sogenannten Warthe-Stadiums hervor, von dem im Schriftum Zweifel gehegt werden, ob es eines von den Stadien der vorletzten Vereisung ist, oder ob es dem maximalen Bereich der letzten Vereisung entspricht (F. ZEuner, W. Szafer 1953) oder vielleicht sogar eine abgesonderte Vereisung ist (B. Malicki, W. Pozaryski).

Ein klassisches Gebiet, in dem das Warthe-Stadium durch P. Woldstedt (1935) festgestellt wurde und wo es seinen Namen erhalten hat, ist Südpolen. Die dortige Situation müßte also für die Auffassung der diesen Namen tragenden Einheit maßgebend sein.

Vom allgemeinen Standpunkt aus wird die Alterseingliederung des Warthe-Stadiums vor allem durch zwei Tatsachen bestimmt.

Erstens: seine Frontalmoränen liegen ca. $50-120 \mathrm{~km}$ nördlich von der maximalen Vereisung Mittelpolens (Riß, Saale-Vereisung). Ihre Geschiebemergel bedecken Sedimente des vorletzten Interglazials (M/R, Holstein) mit paläobotanisch genau untersuchten Profilen (Olszewice, Barkowice Mokre, Sewerynów, Maków Mazowiecki etc.), deren Alter unbezweifelbar ist (Abb. 1).

Andererseits sind in abflußlosen Vertiefungen auf der Akkumulationsfläche der warthestadialen Geschiebemergel Sedimente des letzten Interglazials (R/W, Eem, Masovien II) in Szeczerców, Dzbanki Kościuszkowskie, Józefớw bei Lodz, Rogôw, Gồra Kalwarii u. a. bekannt.

Die palynologischen Profile dieser Vorkommen wurden überprüft oder wieder bearbeitet. Im klimatischen Optimum zeigen sie ein deutliches Maximum von Corylus und andere Merkmale der Flora, die zweifellos das letzte Interglazial kennzeichnen (R/W, Eem).

Interglaziale Serien desselben Alters und mit ähnlichen palynologischen Profilen sind auch im Süden, außerhalb des Bereiches des Warthe-Stadiums bekannt, wo sie sich gleichfalls in abflußlosen Vertiefungen im Hinterland der Frontalmoränen Mittelpolens (Bedlno, Sławno bei Radom u. a.) befinden.

Es ist also unbezweifelbar, daß sich das Warthe-Stadium zusammen mit dem vorhergehenden mittelpolnischen Stadium zwischen dem vorletzten $(M / R)$ und dem letzten $(\mathrm{R} / \mathrm{W})$ Interglazial befindet. 


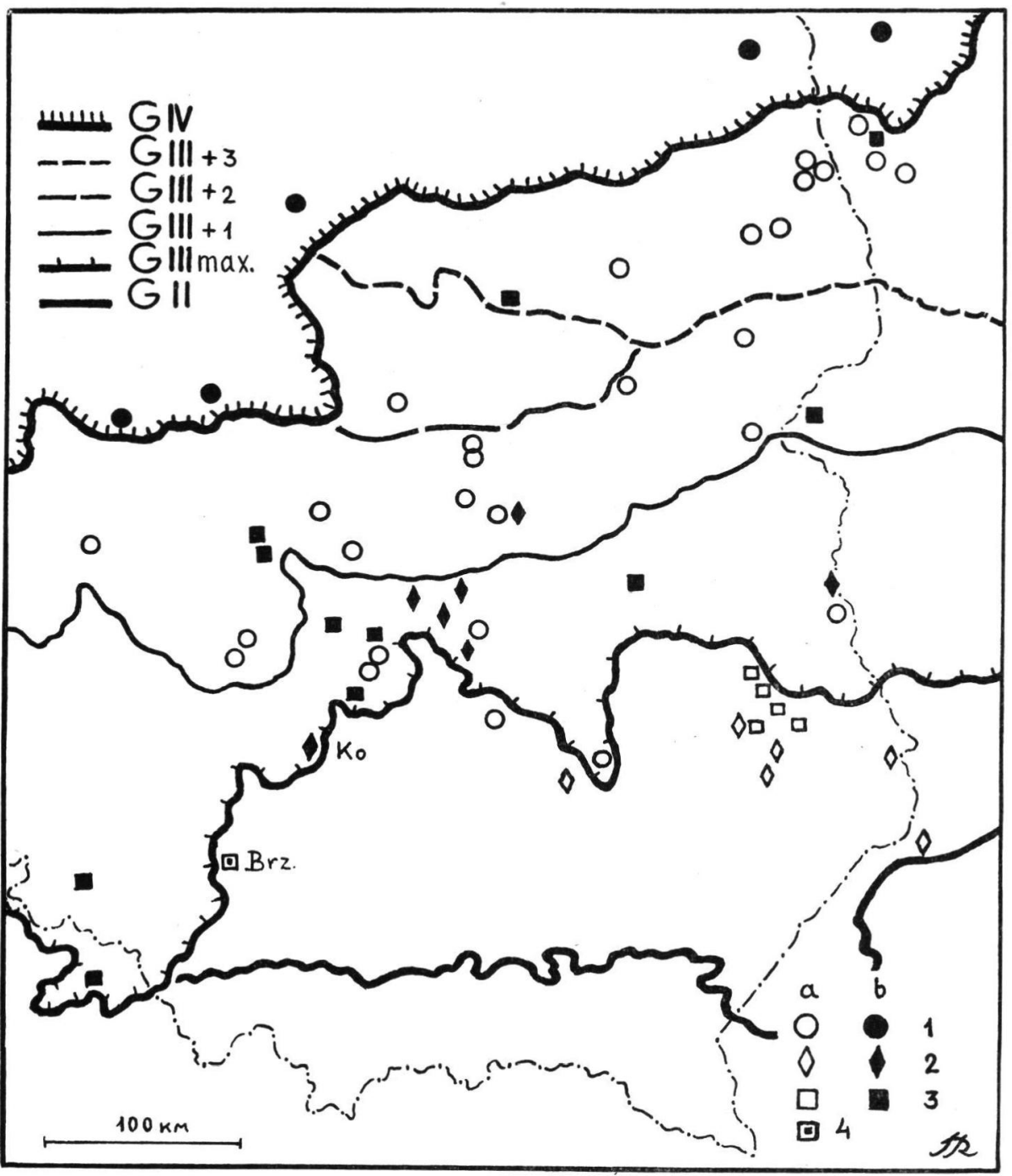

Abb. 1. Vorkommen organogener Sedimente des letzten und vorletzten Interglazials.

1. Das letzte Interglazial (Eem)

2. Die Fundorte der Flora aus der Transgressionsperiode der Mittelpolnischen Vereisung.

3. Das "Große" Interglazial (Holstein)

4. Die Fundorte der Flora aus dem Pilica-Interstadial (Präwarthe-Interstadial).

$\mathbf{a}=$ die durch die Moränenformationen der Mittelpolnischen und die letzten Vereisung nicht bedeckten,

$\mathrm{b}=$ die davon bedeckten Vorkommen.

Angegeben ist der Bereich der letzten (G IV) und der Mittelpolnischen (G III) Vereisung im Radomka (max.)-, Warthe (+1)-, Wkra (+2)- und Mława (+3)-Stadial und der Bereich der Krakauer Vereisung (G II).

Infolgedessen fällt gänzlich die Hypothese aus, daß das Warthe-Stadium eine gewisse Verbindung mit der letzten Vereisungsperiode habe. Es bleiben nur die zwei folgenden Ansichten. Um sie zu erklären, müssen wir uns mehr in die einzelnen Probleme der Stratigraphie dieses Teiles des Pleistozäns vertiefen. 

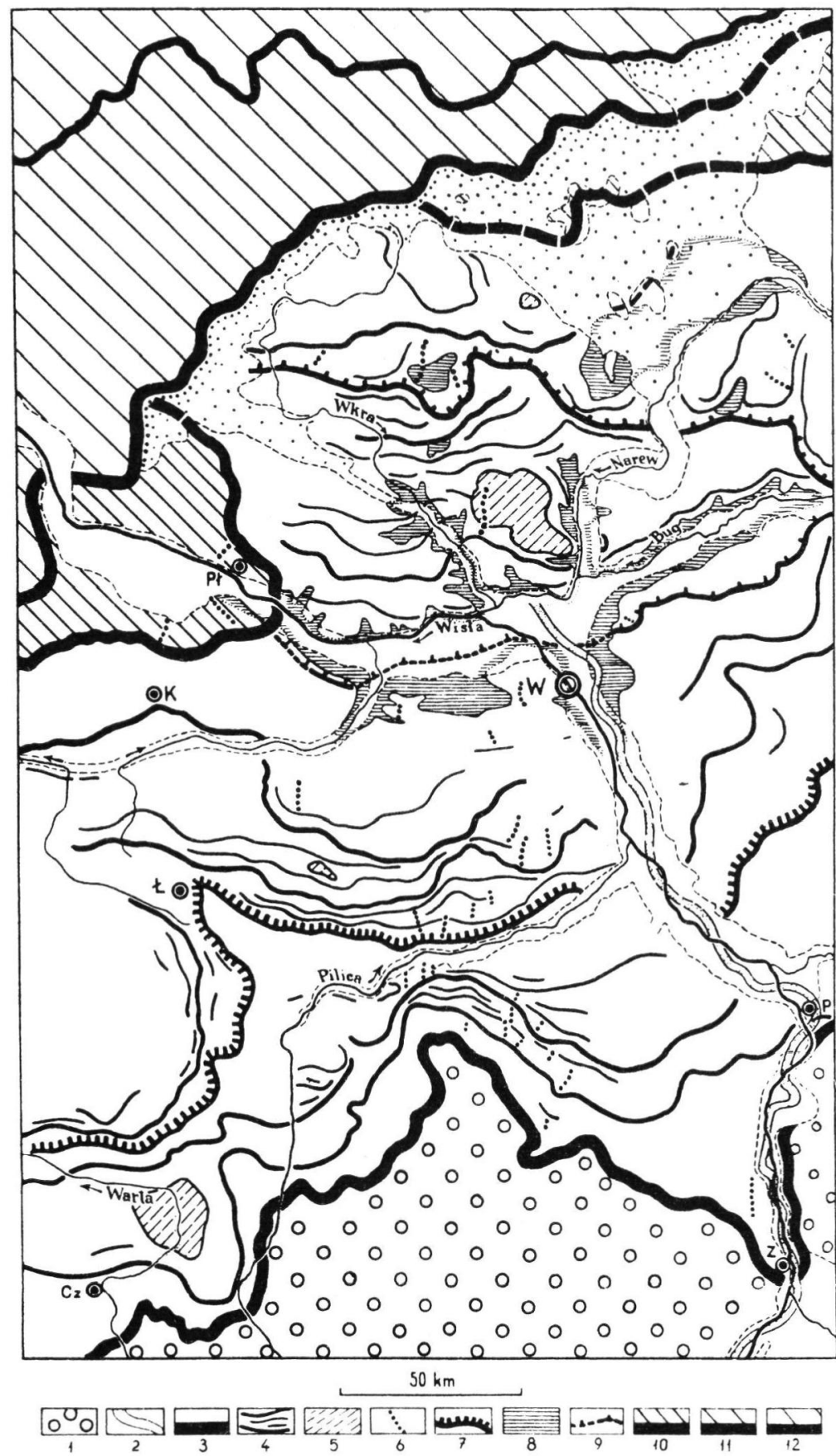

Abb. 2. Der Verlauf der Rezession der Mittelpolnischen Vereisung (Riss).

1. Das periglaziale Gebiet der Mittelpolnischen Vereisung.

2. Das Tal des tiefsten Einschnitts der Weichsel während des "Großen Interglazials" ("MindelRiss").

3. Der maximale Bereich der Mittelpolnischen Vereisung . 
Infolge des großen Zwischenraumes, der die Endmoränenzone Mittelpolens von der Warthe-Endmoränenzone und diese auch sehr breite Zone $(120-200 \mathrm{~km})$ von dem maximalen Bereich der letzten Vereisung (Baltische Vereisung, Nordpolnische Vereisung, Vistulian, Weichsel-Eiszeit, Würm) trennt, und infolge davon, daß sie durch die später eingedrungenen Gletscher nicht bedeckt und glazitektonisch nicht gestört wurden, haben wir in Mittelpolen außerordentlich günstige Bedingungen, um im Gelände den ununterbrochenen Zusammenhang der Niveaus der Geschiebemergel und die sukzessiven Rezessionsphasen zu untersuchen. Für diesen Zweck sind auch zahlreiche Bohrungen wichtig, ferner eine Reihe von großen Aufschlüssen, die eingehende stratigraphische und paläomorphologische Studien ermöglichen.

Daraus ergibt sich das folgende Bild von jener Periode, die mit dem Augenblick beginnt, als die mittelpolnische Vereisung (Riß, Saale) ihren maximalen Bereich erreicht hatte (Abb. 2):

Die mittelpolnische Vereisung (Riß, Saale-Vereisung) reicht mit ihrer maximalen Ausdehnung bis an den äußeren Fuß des Mittelgebirges (Góry Świetokrzyskie), bis zum Fuß des Polnischen Juras und zu der Hochebene von Lublin. Das Inlandeis überwindet steilere Hänge dieser Anhöhen nicht mehr und dringt nur stellenweise etwas in die Täler hinein, deren Ausgänge der Bewegungsrichtung des Eises entsprechen. Dagegen reichte es in dem tiefer gelegenen Gelände zwischen den oben erwähnten Anhöhen viel weiter nach Süden, und bildet den großen Radomer Lobus, der den Weichseldurchbruch schließt und bis Zawichost bei Sandomierz reicht, zwei kleinere Loben zwischen dem Mittelgebirge und dem Polnischen Jura (die Loben von Końskie und von Koniecpol) und die große schlesische Zunge, die am weitesten nach Süden, bis zur Mährischen Pforte, vorrückt.

Diese maximale Phase (die Glaziphase von Gowarczów; G III max. a) des Glazistadiums vom größten Bereich der mittelpolnischen Vereisung dauert jedoch nicht lange und bringt nur unbedeutende Formen einer Endmoränen-Akkumulation hervor, obwohl sie eine ziemlich gut ausgebildete Geschiebemergelschicht hinterläßt. Viel bessere Merkmale hat die nächste Phase (die Glaziphase von Opoczno; G III max. b), deren Bereich in vielen Stellen gut entwickelte, hohe Endmoränenwälle und ein neues Geschiebemergelniveau bestimmen. Die Zweiteilung dieser Geschiebemergel ist noch bis $10-15 \mathrm{~km}$ im Hinterlande der Endmoränen der Opoczno-Glaziphase bemerkbar. Noch weiter dahinter kommen sie zusammen und bilden ein einziges Niveau.

Man kann daraus den Schluß ziehen, daß im Westen des Kreises Radom (wo man es am genauesten beobachten konnte) nach der maximalen Phase (G III max. a) eine Rezession des Inlandeises von ca. $20-25 \mathrm{~km}$ und dann eine Transgression von $10-15 \mathrm{~km}$ stattgefunden hat.

Diese Schwankung ist also vom Range einer Intereis-Phase (die Wolanów-IntereisPhase; G III max. a/b). Sie ist charakterisiert durch die Sedimente, welche die Geschiebemergel der oben erwähnten zwei Glaziphasen (G III max. a; G III max. b) trennen. Wir beobachten hier, bei Berücksichtigung der chronologischen Ordnung, die Spur einer schwachen Erosion (von 5-8 $\mathrm{m}$ ) und dann eine Akkumulation von Sanden und Kiesen und von Warwen.

4. Die Phasen und Stillstands-Etappen der Rezession der Mittelpolnischen Vereisung.

5. Toteis-Gebiete.

6. Oser und ähnliche Formen.

7. Der maximale Bereich des Warthe-Stadials.

8. Der Warwenton des Warschauer Staubeckens und anderer Staubecken, nach dem WartheStadial.

9. Der maximale Bereich des Wkra-Stadials.

10.-12. Die Bereiche der Hauptphasen der letzten Vereisung. Abkürzungen der Städtenamen: $\mathrm{C} z=$ Czestochowa, $\mathrm{K}=$ Kutno, $\mathrm{E}=$ Eódz, $\mathrm{P}=$ Puławy, $\mathrm{Pl}=$ Płock, $\mathrm{W}=$ Warschau, Ko $=$ Koniecpol, $\mathrm{Brz}=$ Brzozowica bei Bedzin . 
Die folgende Rezession von der Linie der Endmoränen der Opoczno-Glaziphase (G III max. b) umfaßt 30-35 km. Sie durchläuft mehrere Etappen, die durch die Formen der Endmoränen-Akkumulation bestimmt werden, die jedoch keine Geschiebemergelniveaus bilden. Auf dem unbedeckten Teil der Akkumulationsoberfläche hat man, außer einer maximalen, noch drei Stillstands-Etappen der Front des Inlandeises feststellen können. Es ist möglich, daß es deren noch mehr gibt, aber sie sind unter der Decke verschwunden, die das nächste Vorrücken des Inlandeises hinterlassen hat (Odrzywół-Glaziphase; G III max. c).

Die Rezession nach der Opoczno-Glaziphase reicht weiter als die vorhergehende und beträgt im Westen des Kreises Radom ca. $50 \mathrm{~km}$ (von der Linie des maximalen Bereiches der Vereisung an gerechnet). Die Sedimente aus der von ihr abgetrennten Przytyk-Interglaziphase (G III max. b/c) sind mannigfaltiger und zeugen von einer längeren Pause als die vorhergehende. Die Erosionsformen in der Oberfläche des Geschiebemergels reichen bis zu einer Tiefe von $6-15 \mathrm{~m}$. Sie sind mit sortierten Flußsanden gefüllt. Oberhalb erscheint eine mehrere Meter dicke Schicht eines typischen subaerischen Lösses. Vor seiner Bildung fand eine Transgression der Odrzywół-Glaziphase (G III max. c) statt, mit der Warwenton verbunden war, und oberhalb dessen lag eine dritte Schicht von Geschiebelehm.

Die Sedimente der Przytyk-Interglaziphase (G III max. b/c) kann man unter der Decke der Moränen der Odrzywół-Glaziphase (G III max. c) in einer ca. $20 \mathrm{~km}$ breiten Zone beobachten. Die sie begleitende Rezession (ca. $30-35 \mathrm{~km}$ ) und das spätere Vorrücken (ca. $20-25 \mathrm{~km}$ ) hatten also eine wenig größere Schwingungsweite als während der vorhergehenden Interglaziphase (Wolanów-Interglaziphase; G III max. a/b). Nichtsdestoweniger zeugen sie von einer Klimaschwankung von ähnlichem Range, und ihre Dauer ist wohl auch beinahe dieselbe.

Die Odryzwół-Glaziphase (G III max. c) hat am äußersten Ende ihres Bereiches, nördlich vom Mittelgebirge, wieder gut ausgebildete große Endmoränenwälle, und sie ist von einem neuen System von Osern begleitet. In dem Maße, wie sich diese Moränen vom Mittelgebirge entfernen, entweder gegen Osten (in Richtung der Radom-Ebene) oder gegen Westen (in Richtung des Widawka-Beckens), wo das Inlandeis vor seiner Stirn keine Hindernisse hatte und frei vorwärtsrückte, werden sie kleiner, stellenweise sind sie unterbrochen oder verschwinden, und der Bereich der Glaziphase wird dann nur durch Zonen einer fluvioglazialen Akkumulation gekennzeichnet.

In der Rezessionszone der Odrzywół-Glaziphase kann man gleichfalls drei bis vier Stillstandsetappen unterscheiden, die bis zum Tal der unteren Pilica reichen.

Nördlich vom Pilica-Tal gibt es schon keine Unterteilung der Geschiebemergel mehr, die wir südlich von ihm beobachtet haben. Bis dort reichten die Rezessionen der vorher besprochenen Interglaziphasen nicht mehr. Ihnen allen entspricht vielmehr nun eine gut ausgebildete Geschiebemergelschicht, die man bis nach Warschau und sogar noch weiter gegen Norden beobachten kann. Das Niveau dieser Lehme wird in nördlicher Richtung allmählich niedriger und liegt ziemlich flach; man kann vermuten, daß sie das Zungenbecken des Inlandeises bedeckten, das in späten Etappen der Rezession der OdrzywółGlaziphase entstanden war (G III max. c).

Wir sind also nun schon bis zu dem Augenblick der Rezession gelangt, in dem die Front des Inlandeises ihre Dynamik verliert und die schnell fortschreitende Ablation die passiv liegenden Massen des stagnierenden Eises zerstört. Wir befinden uns demnach an der Schwelle einer wichtigen und größeren Erwärmungsperiode, während der die aktive Front des Inlandeises eine Entfernung von mindestens $200 \mathrm{~km}$ nördlich von der Linie seiner maximalen Ausdehnung in der Zeit der mittelpolnischen Vereisung erreicht hat.

Die ganze obige, vielleicht zu genaue, obgleich nur kurze Schilderung des Verlaufes der Glazistadialrezession der maximalen mittelpolnischen Vereisung (Riß, Saale), ver- 
suchte die Bedeutung des Zurückweichens des Inlandeises während der Erwärmung, die vor dem Vorstoß des Warthe-Stadiums stattfand, darzulegen.

Wir besitzen darüber auch Kenntnisse aus erster Quelle dank den Untersuchungen der Sedimente und der Paläomorphologie aus dieser Zeit.

Die hier erscheinenden Flußablagerungen (bis ca. $20 \mathrm{~m}$ mächtig) vertreten den ganzen Flußakkumulations-Zyklus. Vor ihr fand eine Tiefenerosion statt, die die Flußtäler bis zu 15-20 m unterhalb der Oberfläche des Moränenplateaus, das das Radomka-Glazistadial (G III max.) hinterlassen hat, austiefte. Jetzt ist auch ein ganz gut entwickeltes Fluß-System erkennbar.

Wir kennen die organischen Ablagerungen, die in dem Profil zwischen den Moränen des Radomka- und Warthe-Stadiums liegen, noch nicht; jedoch gibt es auf dem Moränenplateau an dieser Stelle gut entwickelte Niveaus fossiler Podsol-Böden.

Mehr gegen Süden, schon nahe der Peripherie des Bereiches der mittelpolnischen Vereisung, aber ziemlich weit $(80 \mathrm{~km})$ vor der Front des Warthe-Eises in Brzozowica bei Bedzin, erscheint in einer Situation, die höchstwahrscheinlich der uns interessierenden Rezessionsperiode entspricht, eine Torfschicht (S. Gilewska \& L. Stuchlik, 1958), die mit einer Phase verdünnten, subarktischen Waldes beginnt (Kiefern-Birken mit Larix bei 120\% Kräutern - überwiegend Cyperaceae und Artemisia und mit sehr zahlreichen Polypodiaceae). In ihrem mittleren Teil nimmt die Pollenmenge der Kräuter ab, und die palynologischen Spectra melden das Vorhandensein von Kiefern-Fichten-Wäldern (Pinus, Picea) mit einem großen Anteil der Erle (Alnus) und einem unbedeutenden der Hasel (Corylus) und der Weide (Salix). Es erscheinen auch in kleinen Mengen Pollen mehr wärmeliebender Bäume (Tilia, Quercus, Carpinus), die sich jedoch in sekundärer Lagerung befinden oder aus der Ferne herbeigetragen wurden. Unter den Makroresten befanden sich Nadeln und Holz der Fichte (Picea) und des Wacholders (Juniperus), Erlenholz (Alnus), Früchtchen von Sambucus, Samen von Carex und Moosreste (Polytrichum juniperinum). Das Profil wird oben abgeschlossen durch Schichten mit großen Mengen von CyperaceenPollen (bis 208\%) und ziemlich zahlreichen Kieferpollen (vielleicht der Pinus montana?) und ganz wenigen Pollen der Birke (Betula), Fichte (Picea) und Lärche (Larix) (Abb. 3).

Hier ist also eine deutliche klimatische Schwankung vorhanden. Sie beginnt mit einem lichten subarktischen Wald. Im Optimum ist er dicht und hat hauptsächlich Kiefern und Fichten, aber auch Erlen und Hasel.

\section{BRZOZOWICA}

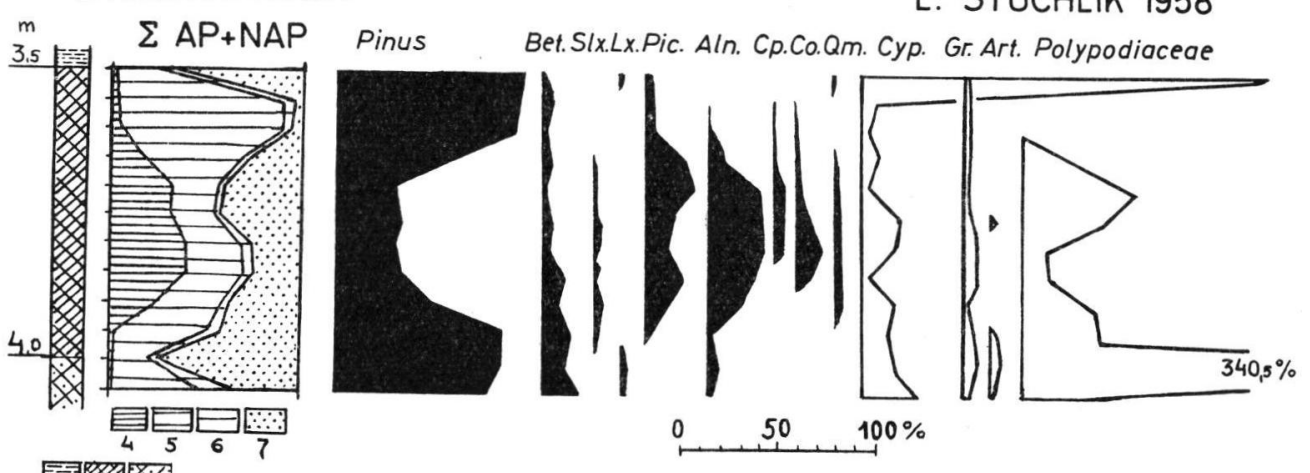

L. STUCHLIK 1958

123

Abb. 3. Das palynologische Spektrum des „Będzin-Interstadials“ (Präwarthe) nach L. STUchlik (aus der Arbeit S. Gilewska und L. Stuchlik, 1958).

Geologisches Profil: 1. Schlamm, 2. Holztorf, 3. Sandiger Torf.

Pollen-Profil: 4. Laubbäume (ohne Betula und Salix), 5. Pinus, 6. Betula, Salix, Larix, 7. NAP. 
Die Waldgrenze verschiebt sich weiter gegen Süden, und dann erscheint von neuem die Tundra, durch ein Kryoturbations-Niveau in den höheren Schichten des Profils und das Vorhandensein von Armeria iversini gekennzeichnet.

Sowohl die Sedimente (ein Zyklus der Tiefenerosion und Flußakkumulation) als auch das palynologische Profil von Brzozowica (eine gemäßigte Erwärmung, mit Kiefer-Fichtenwald im Optimum) und das Vorhandensein von Podsolböden kennzeichnen die Erwärmungsperiode, die der Verfasser für ein Interstadial hält (was er zu beweisen versuchen wird) und „Pilica-Interstadial“ nennt, weil in diesen Zeiten das Tal des unteren Laufes dieses Flusses sich zu bilden anfing.

Am Ende dieser Periode erscheinen lößartige Lehme und ein typischer subaerischer Löß, und zwar unter den Geschiebemergeln des Warthe-Stadiums in der Gegend von Warka an der Pilica (W. Karaszewski 1952, Z. Lamparski 1961) und weiter gegen Norden bei der Góra Kalwarji (Kalvarienberg), die $25 \mathrm{~km}$ südlich von Warschau liegt. Anderswo findet man im jüngeren Teil der fluviatilen Serie stellenweise Warwen und fluvioglaziale Sande, die mit dem wieder vorrückenden Inlandeis verbunden sind. Dieses kam wahrscheinlich ziemlich schnell und ohne große Unterbrechungen vorwärts, jedenfalls erlauben die bekannten Profile nicht festzustellen, daß in der Lage der Eisfront Schwankungen stattgefunden haben.

Diese neue Transgression, die bereits zum Warthe-Stadium gehört, gelangt zur unteren Pilica, erfüllt das Becken der mittleren Warthe und hat fast genau denselben Verlauf, den P. Woldstedt im Jahre 1935 auf seiner Karte gezeichnet hat. Óstlich der Weichsel gelangt sie bis zum südlichen Poglasie und nähert sich dem Bug unweit von Mielnik.

Wo man die Rezession des Pilica-Interstadials (G III + max./+1) feststellen konnte, zeigt sich, daß während der folgenden Transgression die Eisfront um mindestens 100 bis $150 \mathrm{~km}$ vorgerückt ist. Zu den Randmoränen der mittelpolnischen Vereisung blieben durchschnittlich $50-100 \mathrm{~km}$ und nach Schlesien hin sogar bis $200 \mathrm{~km}$.

Im Pilica-Interstadial hatte sich ein Gewässersystem gebildet, in welchem der Abfluß vorwiegend in östliche Richtung gegangen war, und zwar in das Stromgebiet des Dniepr (durch die Täler der Pilica, des unteren Wieprz, der Krzna, des Michawiec und der Pripet, und an das sich die schon bestehenden und dem heutigen System ähnlichen Flußgebiete der oberen Warthe, oberen Weichsel und des oberen Bug angeschlossen hatten). Dieses Abflußsystem wurde durch das Eindringen des Inlandeises des Warthe-Stadiums im allgemeinen nicht verletzt. Zerstört wurde nur der linke, nördliche Teil dieses Abflußsystems. An seine Stelle kamen die Abflüsse der Schmelzwässer aus dem Inlandeis, das im Sommer mehr Wasser lieferte als vorher die Flüsse. Die Flüsse (Pilica, der untere Wieprz, die Krzna, der Pripet) verwandelten sich in einen großen Strom, der auf einer Strecke von mehreren hundert Kilcmetern alle Abflüsse aus dem Randteil des Inlandeises, östlich vom Meridian $19^{\circ} \mathrm{E}$, sammelte.

Die Endmoränenzone, die dem maximalen Bereich des Warthe-Stadiums entspricht, ist am besten entwickelt entlang der Wasserscheide zwischen dem obenerwähnten östlichen (Pilica-Krzna-Pripet) und dem westlichen Zusammenfluß (obere Oder-Schwarze Elstermittlere Elbe), wo sich diese Wasserscheide am meisten dem südlichen Hochgelände nähert (nördlicher Teil des Polnischen Juras). Sie ist ziemlich gut auch noch etwas weiter östlich der Weichsel zu verfolgen. Jedoch bilden sich schon morphologisch in Südpodlasien weniger ausdrucksvolle und schwächer akzentuierte Formen. Loben.

Die Eisfront dieses Stadiums teilt sich deutlich in eine Reihe großer, regelmäßiger

Im Vergleich zu den mittelpolnischen Moränen hebt sich in den Moränen des WartheStadiums ein großer Unterschied in der Zusammensetzung der begleitenden Geschiebe hervor. Die mittelpolnischen Moränen, besonders aus den älteren Phasen des RadomkaStadials (G III max. a, G III max. b) kennzeichnet ein sehr hoher Anteil von Gesteinen 
lokaler Herkunft (bis $80 \%$ der Fraktion $0,5-5 \mathrm{~cm}$ ) und ihre große regionale Vielfalt, die von einer bedeutenden Exarationskraft in der Randzone zeugt. Es wird dies auch bestätigt durch das Auftreten von Rundhöckern (Roches moutonnés) in der Gegend von Czestochowa, und zwar auf den aus massiven Kalksteinen des oberen Juras aufgebauten Hügeln. Der Anteil des lokalen und des skandinavischen Materials in diesen Moränen ist im einzelnen sehr verschieden und läßt sich nicht in charakteristischen Koeffizienten ausdrücken. Es ändert sich auch sehr die Menge der Feuersteine (meist aus dem oberen Jura), die ebenfalls durch den Gletscher aus dem örtlichen Untergrund mitgenommen wurden. Ahnliche und nicht geringere Überraschungen bereiten die südbaltischen paläozoischen Kalksteine (Gotlandium und Ordovicium), welche im allgemeinen nicht zahlreich sind, die jedoch stellenweise in sehr großen Mengen auftreten. Wahrscheinlich steht das im Zusammenhang mit einer sekundären Zumischung der älteren Geschiebemergel (Elster, Mindel), die in die Moränen der folgenden Vereisung aufgearbeitet wurden.

Interessante Änderungen zeigen sich auch in der Zusammensetzung der skandinavischen Geschiebe. Diese Änderungen sind jedoch nicht so einfach, wie V. und K. Milthers es glaubten (1938). Man sieht das am besten bei der Verteilung der baltischen Porphyre, die diese Autoren als leitend für ihre "Warthe-Vereisung“ angenommen haben (Abb. 4).

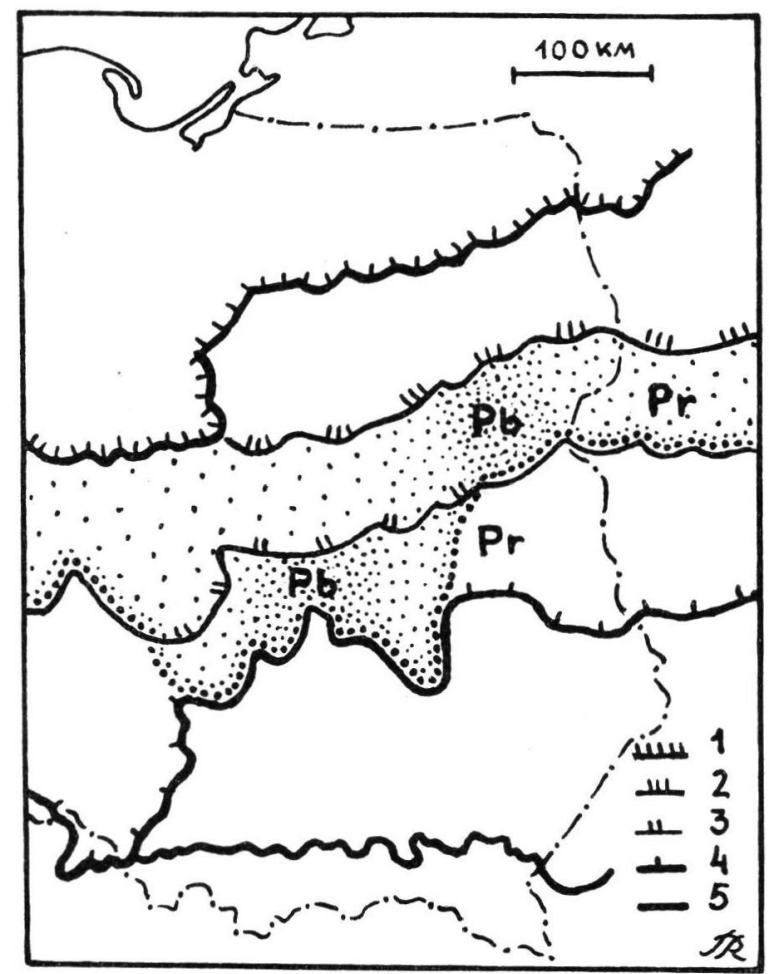

Abb. 4. Die Verteilung der Baltischen Braunen Porphyre in den Moränen der Mittelpolnischen Vereisung (weit punktiert) und ihr häufigstes Erscheinen (dicht punktiert).

$\mathrm{Pb}, \operatorname{Pr}=$ Die Lage der Sektoren der maximalen Häufigkeit der Baltischen Braunen $(\mathrm{Pb})$ und Roten (Pr) Porphyre im Radomka (maximalen)- und im Warthe-Stadial.

1. Bereich der letzten Vereisung.

2. Bereich des Wkra- und Mława-Stadials.

3. Bereich des Warthe-Stadials.

4. Bereich des Radomka-Stadials (maximaler Bereich der Mittelpolnischen Vereisung).

5. Bereich der Krakauer Vereisung. 
Heute, nach der Umrechnung ihrer Angaben auf einzelne Sektoren, gesondert für jede Moränenzone, sieht man recht deutlich, daß die Verteilung des Baltischen Braunen Porphyrs in Polen keinen Zusammenhang mit dem Bereich des Warthe-Stadiums hat, sondern im Sektor zwischen $19^{\circ}$ und $22^{\circ} \mathrm{E}$ die Randlage dieses Stadium überschreitet und zu den Endmoränen des maximalen Radomka-Stadiums zieht (G III max.).

Die regionalen Mittel, umgerechnet für größere Gruppen der Stellen, wo die Zusammensetzung der Geschiebe bestimmt wurde, weisen sogar auf eine gewisse Regelmäßigkeit in der Zahl der Verteilung der Baltischen Porphyre, die in den zentralen Sektoren ihres Auftretens deutliche Maxima erreichen. Sie sind in denselben Sektoren für den Roten und Braunen Baltischen Porphyr nicht gleich; gewöhnlich befindet sich der Braune Porphyr mehr im Westen als der Rote, konform mit der Verteilung ihrer Heimatgebiete zwischen den Inseln Gotland und Åland. Diese Maxima der Häufigkeit der beiden Porphyre in Bezug auf ihre gegenseitige Lage rücken während des Warthe-Stadiums bedeutend nach Osten und zeigen eine ziemlich große Änderung in der allgemeinen Richtung der Eismassenbewegung.

Was den Braunen Baltischen Porphyr im Radomka-Glazistadium (G III max.) anbelangt, so fällt das Maximum seines Auftretens auf den Westen des Mittelgebirges (zwischen $20^{\circ}-21^{\circ} \mathrm{E}$ ); im Warthe-Stadium liegt es ca. $200 \mathrm{~km}$ mehr nach Osten (zwischen $23^{\circ}-24^{\circ} \mathrm{E}$ ). In ähnlicher Weise verlagert sich das Maximum des Roten Baltischen Porphyrs von der Gegend des unteren Wieprz $\left(21^{\circ} 30^{\prime}-23^{\circ} \mathrm{E}\right)$ auf das rechte Bug-Ufer $\left(25^{\circ}-26^{\circ} \mathrm{E}\right)$.

Die Rezession des Warthe-Stadiums zeigt ebenso wie im Radomka-Stadial in seiner Randzone zwei deutliche Phasen (die Phase von Warka, GIII+1a, und die Phase von Grójec, G III + 1b), die durch das Intervall von Rawa $(G I I I+1 a / b)$ getrennt werden. Ihr Bereich zeichnet sich durch schöne Endmoränen-Züge aus. Mit jeder dieser Linien ist ein anderes System von Osern und ein anderes Abflußnetz der Schmelzwässer verbunden. Innerhalb jeder dieser Phasen kann man ein paar Rezessions-Stillstandsetappen unterscheiden.

Nach dem Überschreiten der Linie Nadarzyn-Zyrardów-Skierniewice-Zgierz etc. nimmt die Dicke der Eisakkumulation des Warthe-Stadiums ab, die Formen der EndmoränenAkkumulation verschwinden, und dieses ganze Stadial ist nur durch ein nicht sehr dickes Geschiebemergelniveau vertreten, das man bis zum nördlichen Masovien beobachten kann, $\mathrm{da}$ es ein besonderes Moränenniveau bildet.

Innerhalb der einzelnen Loben des Inlandeises liegen Zungenbecken (Widawka-Becken, Warschauer-Becken u. a.).

Die Fortsetzung der vorletzten Vereisung (Riß, Saale-Vereisung) kann man noch auf dem Gebiet Masoviens beobachten, wo es durch die Ablagerungen der letzten Vereisung nicht bedeckt wurde, so wie es westlich des Meridians $20^{\circ} \mathrm{E}$ geschieht.

Man kann hier feststellen, daß nach dem Warthe-Stadium das Flußnetz wieder lebendig wird. Die vom Süden kommenden Flüsse verlängern ihren Lauf gegen Norden und werden durch den breitenparallelen Hauptstrom gesammelt. Diesmal fließt er nach Westen und vereinigt den Lauf des unteren Bug und der mittleren Weichsel (von Warschau bis Thorn) und hat weiter beinahe dieselbe Richtung wie das spätere Urstromtal der Netze (Thorn-Eberswalder Urstromtal).

Die Flußtäler erreichen eine Tiefe von $20-25 \mathrm{~m}$ und bilden ein gutes Entwässerungssystem. Nachdem sie ihre maximale Tiefe erreicht haben, fangen diese Täler, ebenso wie im Pilica-Interstadial (G III + max./+1), an, sich mit Alluvien zu füllen, die einen Flußsedimentationszyklus bilden und schließlich feinkörnige, horizontal geschichtete Sande zeigen, die sich wohl bereits in Staubecken anhäuften.

Es ist das erste Anzeichen, daß der ziemlich weit im Norden liegende Hauptabfluß des Stromes Hindernisse angetroffen hat oder sogar abgesperrt wurde. Die Ursache dessen ist 
zweifellos eine neue Transgression des Inlandeises, das sich im Optimum des Interstadials hinter den Parallelkreis $52^{\circ} \mathrm{N}$ zurückgezogen und sich nun wieder in Bewegung gesetzt hat und in das oben erwähnte Urstromtal eingezogen ist, das die Wasser nach Westen abführte. Daß es tatsächlich eine Transgression des Inlandeises gewesen ist, geht daraus hervor, daß in dem oberen Teile dieser horizontale geschichteten feinkörnigen Sande zuerst einzelne lehmige Warwenschichten und später eine ganze Serie von Warwentonen erscheint, die einen beträchtlichen Teil des Warschauer Beckens füllen und in schmalen Streifen in die Täler einer Reihe von Nebenflüssen der Weichsel eintreten.

Die stratigraphische Lage der gut bekannten Warwen des Warschauer Beckens, die jahrelang Gegenstand eines Zwistes zwischen J. Lewiński (1921), J. SAMsonowicz (1927) und S. LENCEwICZ (1927) gewesen ist, wurde neuerdings in einer verhältnismäßig einfachen Weise entschieden, die die Ansichten dieser Autoren miteinander in Einklang bringt.

Tatsächlich haben, gemäß der Behauptung von S. LENCEwicz, die Warwen im nördlichen Teil des Warschauer Beckens eine Geschiebemergeldecke oder deren Residuum; in seinem südlichen Teil dagegen gibt es nach der Meinung von J. Lewiński und J. SAMsonowICZ ganz gewiß keinen Hinweis dafür, daß das Inlandeis auf den Warwen gelegen hat.

Die Verbindung mit den Profilen des nördlichen Masoviens und mit den Moränenzügen auf dem rechten Weichselufer erweist, daß wir es hier mit einer Transgression des Inlandeises zu tun haben, das nur teilweise in das Warschauer Becken eingetreten war und nur seinen nördlichen Teil bedeckt hat.

Es lohnt sich vielleicht noch, an dieser Stelle zu betonen, daß das Warschauer Staubecken, ebenso wie eine ganze Reihe von südpolnischen Staubecken, Veränderungen der Fazies in seinen südlichen Teilen zeigt. Man kann hier ein periodisches Vorrücken der sandigen Deltas von Flüssen, die vom Süden kamen, feststellen. Sie sind durch Warwenschichten mit Trockenrissen (infolge jahreszeitlicher Austrocknung?), durch Spuren einer Erosion oder durch eingelagerte mittel- und grobkörnige Sande gekennzeichnet.

Die Zeit, welche die neue Transgression des Inlandeises, bereits die zweite nach dem Maximum der Vereisung (Wkra-Stadial, G III+2), vom Warthe-Stadium (G III+1) trennt und Bug-Narva-Interstadial (G III +1/2) genannt wird, hat einen ähnlichen Verlauf wie das Pilica-Interstadial (G III $+\max . /+1)$. Wir haben hier, wie im vorhergehenden Fall, eine ähnliche Phase einer ziemlich deutlichen Tiefenerosion der Flüsse und dann einen ganzen Zyklus einer Alluvialakkumulation, mit der Phase eines Wasser- und Staubeckens und beendet mit dem Eindringen des Inlandeises. Dadurch wird die Ansicht bestätigt, daß wir es hier tatsächlich mit einer Einheit vom Range eines Interstadials zu tun haben.

Die Rezessionszone des Wkra-Stadials ist sehr gut im nördlichen Masovien sichtbar. Westlich $20^{\circ}$ östl. L. ist sie vollkommen durch die letzte Vereisung bedeckt. Die hier durchgeführten eingehenden Untersuchungen erlauben nach denselben Grundsätzen wie vorher drei Glaziphasen (von Zakroczym, G III $+2 a$, von Nasielsk, G III $+2 b$ und von Ciechanów, GIII+2c) und zwei Intervalle (von Radzików, G III + 2a/b und von Krubin, $\mathrm{G} I I I+2 \mathrm{~b} / \mathrm{c}$ ) und eine ganze Reihe von indirekten Stillstandsetappen zu unterscheiden.

Nach dem Wkra-Stadial haben wir wieder eine bedeutende Rezession des Inlandeises (Krubin-Interstadial, G III + 2/3) und eine abermalige große Transgression (Mlava-Stadial, G III+3). Diese unterscheidet sich recht deutlich von der vorhergehenden durch eine größere Frische der Formen der glazialen Akkumulation, obgleich es hier keine Seen gibt, die so charakteristisch für das Gebiet der letzten Vereisung sind.

Bis zur letzten Zeit bestanden Zweifel, ob man die "Mlava-Transgression“, die schon durch J. Behr \& O. Tietze (1912) und S. Wollosowicz (1924) beobachtet wurde, zu der letzten (Würm-) oder vorletzten (Riß-)Vereisung zählen muß. Genaue Beobachtungen östlich von Nordmasovien haben erwiesen, daß die durch die letzte Vereisung nicht bedeck- 
ten Torfe des letzten Interglazials (Z. Bокówкo-DŁuzaK \& B. Halicki, 1957) in der Gegend von Łomza (Konopki Leśne), nördlich von dem Endmoränenzuge des Mława-Stadials liegen.

In die Zeit der Rezession des Mława-Stadials (G III+3) muß man auch das schön erhaltene Moränengelände stellen, das nördlich von Bialystok liegt, und dessen Alter durch das letzte Interglazial aus der Gegend von Sidra (K. Bitner, 1956) und Augustów (Z. Bonówкo-DŁuZaK \& B. Halicki, 1957) richtig bestimmt wurde.

Der obige Úberblick des Verlaufes der Rezession der vorletzten Vereisung (Saale-Vereisung, Riß) scheint die Stellung des Warthe-Stadiums als eines der großen Stadiale dieser Vereisung genügend zu zeigen. Es unterscheidet sich nicht besonders von den zwei späteren (Wkra-Stadial, G III+2 und Młava-Stadial, G III +3) und auch nicht von dem vorhergehenden Stadial des maximalen Bereiches (Radomka-Glazistadial, G III max.).

Seine richtige Einstufung wurde zweifellos durch die Tatsache erschwert, daß im westlichen Teil des Mitteleuropäischen Tieflandes die jüngeren Stadiale der vorletzten Vereisung (Saale, Riß) durch eine Transgression der letzten Vereisung bedeckt wurden. In gewissem Grade überraschend könnte auch die Selbständigkeit der Transgression des Warthe-Stadiums scheinen, die kein Pendant während des letzten Eindringens der skandinavischen Gletscher in die südliche Ostseeküste hat. Immer mehr neigt man jedoch zu der Ansicht, daß die während der letzten Kälteperiode stattgefundene Eistransgression nur eine kurze glaziale Episode, nämlich am Ende dieser Periode gewesen war (P. Woldstedt 1958). Diese Episode könnte eher mit den Stadialen der vorletzten und älteren Vereisung als mit diesen ganzen Kälteperioden verglichen werden.

Es mögen Zweifel bestehen, ob das „Będzin-Interstadial“ aus Brzozowica tatsächlich dem Pilica-Interstadial (G III + max./+1) oder vielleicht dem späteren Bug-Narev-Interstadial $(G I I I+1 / 2)$ oder dem Regimin-Interstadial $(G I I I+2 / 3)$ entspricht. Es ist jedoch sicher, daß das Będzin-Interstadial eines der oben erwähnten Interstadiale ist, und daß es die Weite der klimatischen Schwankung charakterisiert, die die großen stadialen Transgressionen der vorletzten Vereisung voneinander trennen.

Der Verfasser unternimmt es nicht, eine Parallele zwischen den durch ihn untersuchten Stadialen Polens und den entsprechenden Einheiten Deutschlands aufzuzeigen, obgleich die Folgerungen, die man ziehen könnte, ziemlich klar zu sein scheinen. Es drängt sich auch die Bemerkung auf, daß in einer Reihe von Fällen die Einheiten, gemäß den durch den Verfasser angenommenen Grundsätzen „Interstadiale“ genannt, nur als Intervalle angesehen werden können. Dementsprechend sind manche „Stadiale“ nur Glaziphasen.

Um etwaige Mißverständnisse zu verhüten, muß noch betont werden, daß dem maximalen Radomka-Stadium noch zwei Stadiale vorhergegangen sind. Das spätere "vormaximale" (G III -1) Stadial am unteren Bug ist durch einen Geschiebemergel charakterisiert, der von einem Eindringen des Inlandeises mindestens bis zu $52^{\circ} 30^{\prime} \mathrm{n} . \mathrm{Br}$. zeugt.

Dieses Inlandeis verursachte einen Aufstau der vom Süden kommenden Flüsse und im Norden das Entstehen eines Staubeckens, im Süden des großen „Sees der unteren Pilica“, der bis zur Gegend von Radom reichte. Das ältere Stadial (das „frühe“ Glazistadial G III -2) im nördlichen Masovien bildete Staubecken mit Warwen, die einen Gletscher ankündigten, dessen Moränen man jedoch in dieser Gegend nicht hat finden können.

Diese kalten Glazistadiale trennen die Perioden ziemlich bedeutender Erwärmungen, die palynologisch in Mittelpolen definiert und als Interstadiale von Witaszyn (G III $-1 /$ max.) und Barkowice ( $\mathrm{G}$ II $-2 /-1$ ) anerkannt wurden.

Diese Frage ist jedoch mit dem Problem der Stratigraphie des vorletzten "großen“ Interglazials (Holstein-Wärmezeit, Mindel/Riß) verbunden und wurde bei einer anderen Gelegenheit besprochen (S. Z. RózYCKI, 1961). 


\section{Bibliographie}

Behr, J. \& Tietze, O.: Die Fortsetzung der Lissaer Endmoränen nach Russisch-Polen und die Endmoränen bei Mława. - Jb. Preuß. Geol. Landesanst. 33, I, 1912.

Borówкo-DŁuzak, Z., \& Halicki, B.: [Interglacial sections of the Suwałki region and of the adjacent territory]. - Acta Geologica Polonica, VII, 4, 1957.

Gilewska, S. \& Stuchlik, L.: [Pre-Warta interstadial at Brzozowica near Bedzin]. - Monographiae Botaniceae 7, 1958.

KofCZyŃSKa-ZANDARSKa, K.: [Development of relief in the Radzików area during the recession of the Middle-Polish glaciation]. - Biuletyn Geologiczny Uniwersytetu Warszawskiego. I, 1, 1961. - - [La stratigraphie et la paléomorphologie du Pleistocène des environs de Wychódzc à l'Est de Wyszogród]. - Prace o pleistocenie Polski Środkowej, vol. II (Acta Geologica Polonia, XIV, 3), 1964.

LAMPARSKI, Z.: [The percentage distribution of local material in end-moraines of the Middle-Polish (Riss) Glaciation.] - Prace o pleistocenie Polski Środkowej, I, 1961.

Lencewicz, St.: [Glaciation et morphologie du bassin de la Vistule moyenne]. - Prace P. I. G., II, 2, 1927.

LewiŃski, J.: Badania hydrogeologiczne okolic Warszawy. - Roboty Publiczne, 1921.

MichalsKa, Z.: [Stratigraphy of the Pleistocene and Paleomorphology of the North-Eastern Part of Mazowsze (Central Poland)]. - Studia Geologica Polonica, VII, 1961a. - - [Age determination of end-moraines in the vicinity of Mława and Przasnysz by stratigraphic and paleomorphological studies]. - Prace o pleistocenie Polski Srodkowej, I, $1961 \mathrm{~b}$.

Milthers, V. \& Milthers, K.: [Die Verteilung einiger wichtiger skandinavischer Leitgeschiebe in einem Teile Polens]. - Biuletyn P.I.G., 5, 1938.

Pozaryski, W.: [The Pleistocene in the Vistula Gap across the Southern Uplands]. - Instytut Geologiczny. Prace, IX, 1953.

Rózycki, S. Z.: Sub-stages of the Great Interglacial Stage. (Summary of the results of the research into the stratigraphy of the Quaternary in Poland.). - Prace o pleistocenie Polski Srodkowej, I, 1961 a. - - Middle Poland. Guide-book of Excursion from the Baltic to the Tatras. - INQUA VIth Congress, Poland, 1961 b. - - [Sytème climato-stratigraphique de la division du Pleistocène]. - Prace o pleistocenie Polski Środkowej, II, (Acta geologica polonica, XIX, 2) 1964.

Ruszcyńska-SzenajCH, M.: [The Quaternary in the neighbourhood of Tomaszów Mazowiecki and Sulejów on the Pilica river]. - Praca o pleistocenie Polski Środkowej, I, 1961. - - [Le Pleistocène aux environs de Wyszogród sur la Vistule]. - Prace o pleistocenie Polski Środkowej, II, (Acta Geologica Polonica, XIV, 3), 1964.

Straszewska, K.: [Stratigraphy of the Pleistocene and Paleomorphology of the region of the Bug river]. - Studia Geologica Polonica (in Druck) 1965.

Samsonowicz, J.: Przewodnik geologiczny po Warszawie i okolicy. Wstep ogólny. - Oddz. Warsz. Kom. Fizjogr. PAN. 1927.

Szafer, W.: [Pleistocene stratigraphy of Poland from the floristical point of view]. - Rocznik Pol. Tow. Geolog., XXII, 1, 1953.

Woldstedt, P.: Norddeutschland und angrenzende Gebiete im Eiszeitalter. - Stuttgart 1950. - Das Eiszeitalter. Bd. II. - Stuttgart 1958.

WoŁŁosowicz, S.: [Sur l'extrémité meridionale du lobe glaciaire de Mława pendant la dernière glaciation]. - P.I.G., Sprawozdania 2, 1924.

Manuskr. eingeg. 10. 3. 1965.

Anschrift des Verf.: Prof. Dr. S. Z. Rózycki, Warszawa 1 (Polen), Ul. Krakowskie Przedmieście 30. 